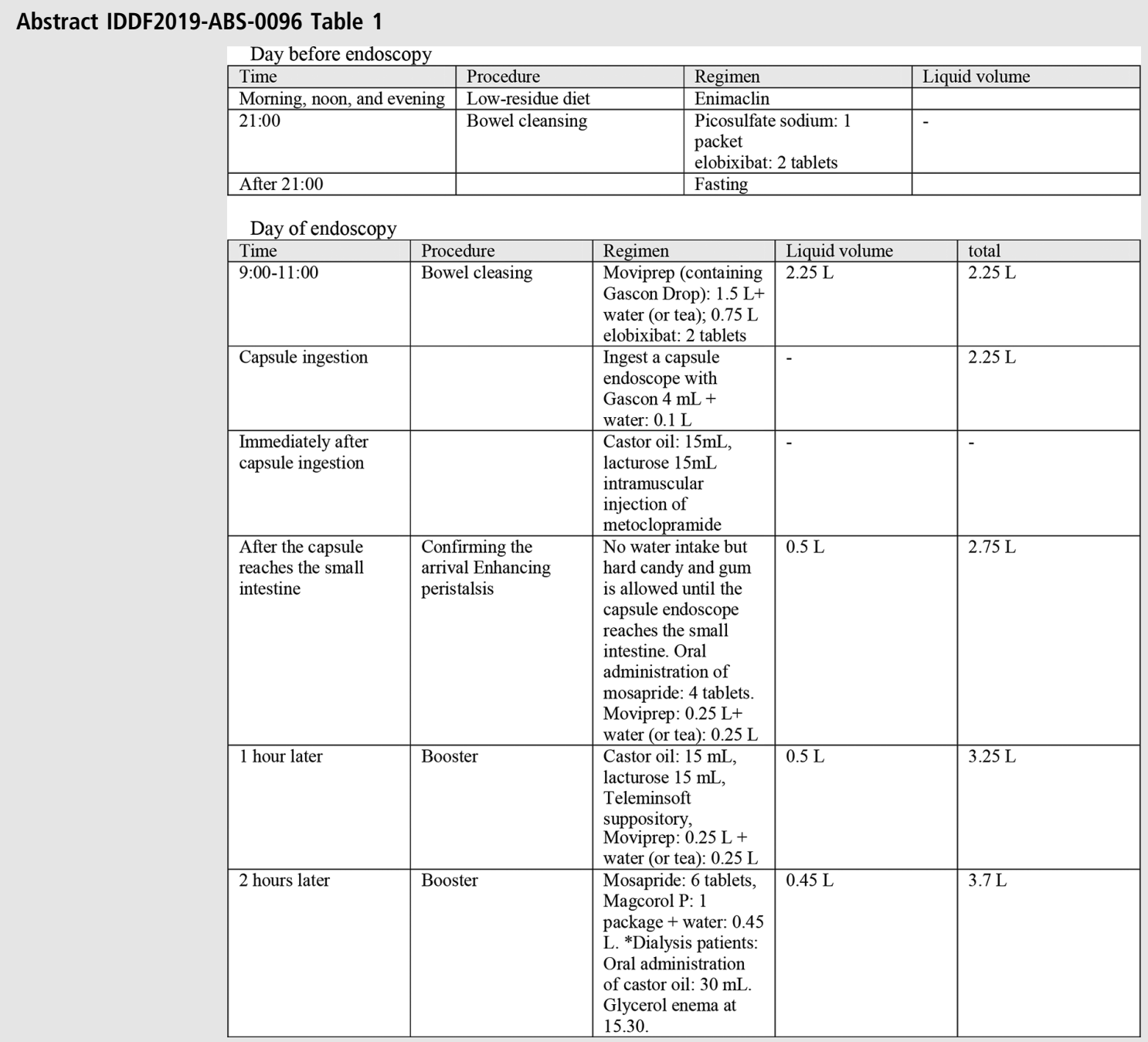

in 2014. For this reason, a detailed examination by capsule endoscopy appears to be useful. However, the capsule excretion rate after recommended bowel preparation reportedly ranges from $70 \%$ to $90 \%$.In this study, we evaluated the efficacy of bowel preparation with Elobixibat and lactulose for improving the capsule excretion rate.

Methods This study included 46 patients who underwent colon capsule endoscopy at Masuko Memorial Hospital since March 2018. The recommended protocol of bowel preparation was modified(with Elobixibat and lactulose) and success rates of completing entire colon observation were compared. Elobixibat(Goofice: EA pharma, Japan) at the beginning of the day before enforcement and the enforcement. Lactulose(Morinaga, Japan) was administered immediately after capsule ingestion and as a booster at one and two hours later. This study was approved by the ethics committee of my hospital, while we obtained written consent from the participants after providing a thorough explanation of the contents and methods of this study. (table 1)

Results The success rate of completing entire colon observation was 43patients were 46(93.4\%)in this study(23 dialysis patients and 23 non-dialysis patients). The success rates were 23 patients included $22(95.6 \%)$ in the non-dialysis patients and $(21 / 23)$ in the dialysis patients.
Conclusions Although the number of patients receiving preparation with Elobixibat and lactulose was small, entire colon observation was successful even in the dialysis patients. Thus, Elobixibat and lactulose appear to be useful for bowel preparation for colon capsule endoscopy.

\section{IDDF2019-ABS-0098 THE EFFECT OF PREDICTABLE NURSING MEASURES ON COMMON BILE DUCT STONE PATIENTS IN POST ERCP COMPLICATIONS}

Fang Wang*, Wen-Hui Tan, Ling He, Fang Lv. Guangdong Second Provincial General Hospital, China

\subsection{6/gutjnl-2019-IDDFabstracts. 142}

Background To explore the effect of predictable nursing measures on preventing post ERCP complications of common bile duct stone patients.

Methods 100 common bile duct stone patients were enrolled in our study. Patients were randomly divided into two groups. Routine nursing was performed in control group $(n=50)$, while predictable nursing was performed in observation group $(n=50)$. Data on overall survival, technical failure, nursing 
satisfaction, complications and days of hospitalization after ERCP were examined and compared.

Results No death occurred in our study. The post ERCP complication rates of observation group were statistically lower than control group $(\mathrm{P}=0.03)$. There were no statistically significant differences in technical failure between the groups $(\mathrm{P}=0.08)$. Technical failure Hospitalization time was decreased and nursing satisfaction was improved markedly in observation group than control group ( $\mathrm{P}=0.01$ and 0.03 respectively).

Conclusions Predictable nursing measures applying to common bile duct stone patients underwent ERCP, helps prevent post ERCP complications, reduce the duration of hospital stays and improve the patients' life quality and satisfaction.

\section{IDDF2019-ABS-0099 SHORT TERM OUTCOMES OF PER ORAL ENDOSCOPIC MYOTOMY (POEM) AS TREATMENT FOR PRIMARY ACHALASIA IN THE PHILIPPINES}

Abigail Valenzuela*, Esperanza Grace Santi. De La Salle University Medical Center, Philippines

10.1136/gutjnl-2019-IDDFabstracts. 143

Background To assess the short term clinical outcome of POEM.

Methods Data on consecutive patients with primary achalasia with epidemiology and clinical response were collected.

Results Twenty-five patients with a mean age of 38 were followed for 2 years. Seventy percent had no previous treatment for achalasia before POEM, 22\% underwent pneumatic balloon dilatation and $8 \%$ had botulinum toxin. The mean baseline Eckardt score was 8.8. Type 2 achalasia was seen in $88 \%$. The mean procedural time was 88.4 minutes. Total myotomy length was $13.5 \mathrm{~cm}$ and the average hospital stay of 5.4 days. Postoperative complication includes capnoperitoneum (16\%). Clinical success was achieved with a post follow up Eckardt score of 0 and an increased BMI from 15 to 19.3 and 19.8 after 6 months and 1 year respectively. All patients had no symptoms recurrence. Four percent had reflux symptoms controlled by PPI. One (4\%) out of 25 patients had endoscopically documented esophagitis after one year.

Conclusions Peroral endoscopic myotomy (POEM) is a minimally invasive procedure for the treatment of achalasia with a promising safety profile and excellent short-term clinical results. The success rate of this study was $100 \%$ with a significant improvement of Eckardt score.

\section{IDDF2019-ABS-0100 LINGUISTIC AND CULTURAL CONSIDERATIONS IN DIAGNOSIS BASED ON PATIENT-REPORTED SYMPTOMS OF FUNCTIONAL DYSPEPSIA IN THAILAND}

${ }^{1}$ Krit Pongpirul ${ }^{*},{ }^{2}$ Michaela Barry, ${ }^{2}$ Amundam Mancho, ${ }^{3}$ Pasa Suksmith. ${ }^{1}$ Chulalongkorn University, Thailand; '2University of Rochester School of Medicine and Dentistry, USA; ${ }^{3}$ Harrow School, UK

10.1136/gutjnl-2019-IDDFabstracts. 144

Background Functional dyspepsia (FD) is a heterogeneous complex of symptoms presenting with effects in the upper gastrointestinal tract. With negative diagnostic workup, the diagnosis is based solely on patient-reported symptoms. As patient-reported symptoms are described variably between different cultures and languages, this study aims to linguistically explore how Thai patients describe functional dyspepsia.

Methods This qualitative study interviewed purposively selected 20 patients diagnosed with functional dyspepsia at outpatient clinics of four different healthcare settings in Thailand. The participants were asked for Thai adjective words that they would use to describe their functional dyspepsia symptoms. The words were then mapped with comparable English terminologies. The words were then compared with the Rome IV Diagnostic Criteria for Dyspepsia.

Results Seven Thai words were revealed from the interviews (frequencies): ปวด ('hurt'), เจ็บ ('ache'), แสบ ('burning/stinging hurt'), จิ๊ด ('sharp/piercing'), บิด ('twist'), จุก ('painful pressure'), and เสียด ('focused pain').

Conclusions Some words used by Thai patients have not been compatible with 'standard' criteria for functional dyspepsia.

\begin{tabular}{|l|l}
\hline IDDF2019-ABS-0101 & ORAL AND TOPICAL APPLICATION OF \\
& KHEAW HOM POWDER ON ORAL ULCER: \\
& EFFICACY AND PATIENT PREFERENCE \\
& (KHOU)
\end{tabular}

${ }^{1}$ Potchara Khamsetha*, 'Pattaraporn Chaihad, 'Subin Kongmuang, 'Nattakitta Pholor, ${ }^{2}$ Krit Pongpirul. ${ }^{1}$ Namphong Hospital, Thailand; ${ }^{2}$ Chulalongkorn University, Thailand

\subsection{6/gutjnl-2019-IDDFabstracts. 145}

Background Kheaw Hom Powder (KHP) is an herbal recipe in the Thai National List of Essential Medicine that comprises of 18 herbs. This prospective observational study investigated the efficacy and patient preference for oral, topical, or both oral and topical preparations.

Methods Participants who met the inclusion criteria were asked to choose one of the following options: oral only (1 g of KHP dispersed in warm water three times daily before a meal), topical only (500 mg of KHP directly applied to the ulcer three times daily after a meal), or both for 6 consecutive days. The diameter of each ulcer was measured at its widest part by using Vernier Caliper (Day 1, 2, and 6). Pain score was assessed by using 10-point Likert's scale (Day 1, 2, and 6). Improvement is defined by $50 \%$ decrease of ulcer diameter or 2 points reduction of pain score. (Thai Clinical Trials Registry No. TCTR20170810003)

Results Fifty participants with a mean age of 54.60 years (female 66\%) were included; of which 60\% had one oral ulcer whereas $34 \%$ and $6 \%$ had two and three ulcers, respectively. The median duration of ulcer at presentation was $\underline{Z}$ days. The ulcers were most common at upper and lower lips (52\%), followed by buccal mucosa (36\%), tongue (14\%), palate $(10 \%)$, and gum $(2 \%)$. The mean pain score of 7.35 (SD 1.29) was reduced to 2.63 (SD 1.93; $\mathrm{p}=0.023$ ) and 0.03 (SD $0.17 ; \mathrm{p}<0.001)$ whereas the mean ulcer diameter of 2.67 (SD 1.75 ) was reduced to 2.22 (SD 1.29; p<0.001) and 1.06 (SD $0.61 ; \mathrm{p}<0.001) \mathrm{mm}$.

Conclusions Kheaw Hom Powder can effectively reduce the pain and the size of oral ulcers. 